ORIGINAL ARTICLE

\section{Identification of Late-Onset Hypogonadism in Middle-Aged and Elderly Men}

\author{
Frederick C.W. Wu, M.D., Abdelouahid Tajar, Ph.D., Jennifer M. Beynon, M.B., \\ Stephen R. Pye, M.Phil., Alan J. Silman, M.D., Joseph D. Finn, B.Sc., \\ Terence W. O’Neill, M.D., Gyorgy Bartfai, M.D., Felipe F. Casanueva, M.D., Ph.D., \\ Gianni Forti, M.D., Aleksander Giwercman, M.D., Ph.D., \\ Thang S. Han, M.D., Ph.D., Krzysztof Kula, M.D., Ph.D., Michael E.J. Lean, M.D., \\ Neil Pendleton, M.D., Margus Punab, M.D., Ph.D., Steven Boonen, M.D., Ph.D., \\ Dirk Vanderschueren, M.D., Ph.D., Fernand Labrie, M.D., Ph.D., \\ and Ilpo T. Huhtaniemi, M.D., Ph.D., for the EMAS Group*
}

A BSTRACT

\section{BACKGROUND}

The association between aging-related testosterone deficiency and late-onset hypogonadism in men remains a controversial concept. We sought evidence-based criteria for identifying late-onset hypogonadism in the general population on the basis of an association between symptoms and a low testosterone level.

\section{METHODS}

We surveyed a random population sample of 3369 men between the ages of 40 and 79 years at eight European centers. Using questionnaires, we collected data with regard to the subjects' general, sexual, physical, and psychological health. Levels of total testosterone were measured in morning blood samples by mass spectrometry, and free testosterone levels were calculated with the use of Vermeulen's formula. Data were randomly split into separate training and validation sets for confirmatory analyses.

\section{RESULTS}

In the training set, symptoms of poor morning erection, low sexual desire, erectile dysfunction, inability to perform vigorous activity, depression, and fatigue were significantly related to the testosterone level. Increased probabilities of the three sexual symptoms and limited physical vigor were discernible with decreased testosterone levels (ranges, 8.0 to $13.0 \mathrm{nmol}$ per liter [2.3 to $3.7 \mathrm{ng}$ per milliliter] for total testosterone and 160 to 280 pmol per liter [ 46 to 81 pg per milliliter] for free testosterone). However, only the three sexual symptoms had a syndromic association with decreased testosterone levels. An inverse relationship between an increasing number of sexual symptoms and a decreasing testosterone level was observed. These relationships were independently confirmed in the validation set, in which the strengths of the association between symptoms and low testosterone levels determined the minimum criteria necessary to identify late-onset hypogonadism.

\section{CONCLUSIONS}

Late-onset hypogonadism can be defined by the presence of at least three sexual symptoms associated with a total testosterone level of less than $11 \mathrm{nmol}$ per liter (3.2 ng per milliliter) and a free testosterone level of less than 220 pmol per liter (64 pg per milliliter).
The authors' affiliations are listed in the Appendix. Address reprint requests to Dr. Wu at the Andrology Research Unit, Developmental and Regenerative Biomedicine Research Group, University of Manchester, Manchester Academic Health Science Centre, Manchester Royal Infirmary, Grafton St., Manchester M13 9WL, United Kingdom, or at frederick.wu@ manchester.ac.uk.

*The members of the European Male Aging Study (EMAS) group are listed in the Appendix.

This article (10.1056/NEJMoa0911101) was published on June 16, 2010, at NEJM.org.

N EnglJ Med 2010;363:123-35.

Copyright () 2010 Massachusetts Medical Society. 
T HE CLINICAL IMPORTANCE OF AN AGErelated reduction in the testosterone level ${ }^{1-3}$ remains controversial. ${ }^{4,5}$ Because of the uncertainty regarding the nature of testosterone deficiency in aging men, ${ }^{6-9}$ recent guidelines have suggested that so-called late-onset hypogonadism be regarded as a clinical and biochemical state with advancing age, characterized by particular symptoms and a low level of serum testosterone. ${ }^{10,11}$ However, few data on hypogonadism in aging men are available $e^{4,8,12}$ because of the lack of evidence regarding the exact criteria for identifying testosterone deficiency in older men who do not have pathological hypogonadism. ${ }^{6,13}$ Although a familiar array of symptoms typify severe testosterone deficiency (total testosterone level, $<6$ to $8 \mathrm{nmol}$ per liter [1.7 to $2.3 \mathrm{ng}$ per milliliter]) in younger patients, symptoms in aging men are nonspecific and are mimicked by other prevalent disorders. The testosterone level below which symptoms of androgen deficiency emerge and adverse health outcomes ensue in older men remains unclear, and the use of arbitrary thresholds is not appropriate. ${ }^{11}$

We conducted a study in a general population of middle-aged and elderly men in order to characterize the clinical symptoms associated with a low testosterone level, to identify the thresholds of testosterone below which such symptoms become increasingly prevalent, and to define essential criteria for the syndrome of late-onset hypogonadism on the basis of the presence of symptoms associated with a low testosterone level. ${ }^{14}$

\section{METHODS}

\section{STUDY POPULATION}

We performed age-stratified, random sampling of men participating in the European Male Aging Study (EMAS) at eight European centers: Manchester, United Kingdom; Leuven, Belgium; Malmö, Sweden; Tartu, Estonia; Lodz, Poland; Szeged, Hungary; Florence, Italy; and Santiago de Compostela, Spain. Men between the ages of 40 and 79 years in population or primary care registers were invited to undergo health assessment by questionnaire, physical and cognitive performance tests, and blood tests for biochemical and hormone measurements. From a total of 8416 invitees, 3369 men (mean age, 59.7 years) were recruited without the use of specific exclusion criteria. The adjusted mean response rate at each center was 43\% (range, 24 to
60). A detailed description of the study design, recruitment, and methods has been reported previously. ${ }^{14}$ Ethics approval for the study was obtained in accordance with local institutional requirements at each center, and written informed consent was obtained from all subjects.

\section{QUESTIONNAIRES}

We collected data regarding sociodemographic and general health status, medical conditions, medications, and lifestyle with the use of questionnaires in local languages that were completed by the subjects. Interviewer-assisted questionnaires that were administered included the Medical Outcomes Study 36-Item Short-Form Health Survey (SF-36) and the Beck Depression Inventory, as described previously, ${ }^{14}$ and the EMAS Sexual Function Questionnaire. ${ }^{15}$

\section{CLINICAL AND LABORATORY ASSESSMENTS}

Height, weight, body-mass index, and waist circumference were measured, as described previously. ${ }^{14}$ A single, fasting venous blood sample was obtained in the morning (before 10 a.m.). Total testosterone was measured by means of gas chromatography-mass spectrometry (GC-MS), as described by Labrie et al. ${ }^{16}$ The lower limit of total testosterone measurement was $0.17 \mathrm{nmol}$ per liter ( $0.05 \mathrm{ng}$ per milliliter). The coefficients of variation of testosterone measurements were $2.9 \%$ within runs and $3.4 \%$ between runs. Sex hormonebinding globulin was measured on the Modular E170 platform immunoassay (Roche Diagnostics), as described previously. ${ }^{17}$ Free testosterone levels were derived from measurements of total testosterone, sex hormone-binding globulin, and albumin. ${ }^{18}$

\section{TRAINING AND VALIDATION SETS}

The cohort was randomly subdivided into a training set and a validation set (Table 1). The associations between selected symptoms and testosterone level were explored in the training set to identify clinical and biochemical criteria for lateonset hypogonadism. The associations between symptoms and a low testosterone level that were identified in the training set were then independently evaluated in the validation set.

In the training set, 32 items from the EMAS questionnaires were considered as possible candidates for symptoms of androgen deficiency on the basis of previous recommendations ${ }^{10,11,13}$ and 
studies $^{19-22}$ (for details, see Section 1 in the Supplementary Appendix, available with the full text of this article at NEJM.org). All items were screened to identify those that were significantly associated with total or free testosterone levels by means of ordinal logistic-regression models (Section 2 in the Supplementary Appendix). Only items that were significantly associated with total or free testosterone levels were selected. Ordinal responses to the selected questions were then dichotomized into symptomatic and asymptomatic categories. The Mann-Whitney test was used to confirm the difference in testosterone levels between the symptomatic group and the asymptomatic group (Table 2, and Fig. 1 in the Supplementary Appendix).

\section{STATISTICAL ANALYSIS}

In the training set, we used locally weighted linear regression ${ }^{23}$ to identify threshold levels of testosterone below which the probability of a symptom increased above the background prevalence in the overall study population. Multiple logisticregression models were used with linear-spline functions ${ }^{24}$ for testosterone levels with adjustment for age and study center, to further define testosterone threshold levels at which a significant change in the likelihood of the symptom occurred.

Multiple correspondence analyses ${ }^{25}$ were carried out in the training set to map associations between categorical variables (the presence or absence of symptoms and a low or normal testosterone level) in a two-dimensional space. Close proximity and similar directionality of variables graphically (from the intersection point of the two axes) indicated a clustering of syndromic association. Multiple correspondence analyses were then performed in the validation set to ascertain whether the clustering in the training set could be confirmed.

As a means of integrating the various criteria and thresholds that were developed and refined in the training set, we used logistic-regression models to quantify the strength of associations between the combination of selected symptoms and testosterone levels below defined thresholds. These models were then applied to the validation set to determine whether the quantified associations between symptoms and low testosterone levels could be independently confirmed. We used the replicable associations between specified combi- nations of symptoms and testosterone thresholds in the validation set as the basis for specifying the minimal criteria for the syndrome of late-onset hypogonadism. As an additional internal validation of the results, we used resampling techniques to derive bootstrap confidence intervals for odds ratios. The possible confounding effects of age, body-mass index, and the number of coexisting illnesses on the association between symptoms and a low testosterone level were assessed by multiple logistic-regression analysis.

\section{RESULTS}

\section{STUDY POPULATION}

From a total of 3369 study participants, 150 were excluded because of known pituitary or testicular diseases or current use of medications that could affect pituitary or testicular function or sex-steroid clearance. These exclusions left 3219 men in the analysis sample. The characteristics of these men, subdivided into training and validation sets, are shown in Table 1.

\section{ASSOCIATIONS BETWEEN SYMPTOMS AND TESTOSTERONE LEVELS}

From an initial pool of 32 candidate symptoms of testosterone deficiency that were explored in the training set, 9 symptoms were confirmed to be related to the total or free testosterone level, with significant differences between the symptomatic group and the asymptomatic group (Table 2). These symptoms included three sexual symptoms (decreased frequency of morning erection, decreased frequency of sexual thoughts, and erectile dysfunction), three physical symptoms (an inability to engage in vigorous activity [e.g., running, lifting heavy objects, or participating in strenuous sports], an inability to walk more than $1 \mathrm{~km}$, and an inability to bend, kneel, or stoop), and three psychological symptoms (loss of energy, sadness ["downheartedness" on questionnaire], and fatigue).

The probability of symptoms increased with decreased levels of testosterone (Fig. 1). The thresholds for total testosterone were approximately $8 \mathrm{nmol}$ per liter (2.3 ng per milliliter) for a decreased frequency of sexual thoughts, $8.5 \mathrm{nmol}$ per liter (2.5 ng per milliliter) for erectile dysfunction, $11 \mathrm{nmol}$ per liter (3.2 ng per milliliter) for a decreased frequency of morning erections (Fig. 1A), and $13 \mathrm{nmol}$ per liter (3.7 ng per milliliter) for diminished vigor (Fig. 1C); free testos- 
terone thresholds for the three sexual symptoms were 160,280 , and 280 pmol per liter $(46,81$, and 81 pg per milliliter), respectively (Fig. 1B), with a threshold of 160 pmol per liter for both sadness and fatigue (Fig. 1F). No thresholds were identified for physical symptoms associated with free testosterone (Fig. 1D) or psychological symptoms associated with total testosterone (Fig. 1E). The probability of symptoms was relatively high $(>0.25)$, even though testosterone was in the unequivocally normal range (Fig. 1A). Only seven of the men had total testosterone levels of more than $35 \mathrm{nmol}$ per liter (10.1 ng per milliliter), which may have given a spurious impression of changes in the probability of erectile dysfunction with high total testosterone levels (Fig. 1A).

\begin{tabular}{|c|c|c|}
\hline Variable & $\begin{array}{l}\text { Training Set } \\
(\mathrm{N}=1610)\end{array}$ & $\begin{array}{l}\text { Validation Set } \\
\text { (N=1609) }\end{array}$ \\
\hline \multicolumn{3}{|l|}{ Age and anthropometric measures } \\
\hline Age $-y r$ & $59.7 \pm 0.3$ & $59.7 \pm 0.3$ \\
\hline Height $-\mathrm{cm}$ & $173.8 \pm 0.2$ & $173.7 \pm 0.2$ \\
\hline Weight — kg & $83.4 \pm 0.4$ & $83.8 \pm 0.4$ \\
\hline Body-mass index' & $27.6 \pm 0.1$ & $27.8 \pm 0.1$ \\
\hline Waist circumference $-\mathrm{cm}$ & $98.1 \pm 0.3$ & $98.8 \pm 0.3$ \\
\hline \multicolumn{3}{|l|}{ Health status } \\
\hline \multicolumn{3}{|l|}{ SF-36 score (high score favorable) } \\
\hline Physical functioning (range, 10-30) & $27.2 \pm 0.1$ & $27.2 \pm 0.1$ \\
\hline Physical role (4-20) & $17.3 \pm 0.1$ & $17.2 \pm 0.1$ \\
\hline Body pain (2-12) & $9.3 \pm 0.1$ & $9.4 \pm 0.1$ \\
\hline General health (5-25) & $18.0 \pm 0.1$ & $17.9 \pm 0.1$ \\
\hline Vitality (4-20) & $14.9 \pm 0.1$ & $15.1 \pm 0.1$ \\
\hline Social role $(2-10)$ & $9.0 \pm 0.0$ & $9.0 \pm 0.0$ \\
\hline Emotional role (3-15) & $13.4 \pm 0.1$ & $13.4 \pm 0.1$ \\
\hline Mental health (5-25) & $20.4 \pm 0.1$ & $20.4 \pm 0.1$ \\
\hline Score on Beck Depression Inventory (low score favorable; range, 0-63) & $6.9 \pm 0.2$ & $6.8 \pm 0.2$ \\
\hline \multicolumn{3}{|l|}{ Hormone levels: } \\
\hline \multicolumn{3}{|l|}{ Testosterone } \\
\hline Total — nmol/liter & $16.5 \pm 0.2$ & $16.6 \pm 0.2$ \\
\hline Free - pmol/liter & $293.0 \pm 2.2$ & $291.6 \pm 2.2$ \\
\hline Sex hormone-binding globulin - nmol/liter & $42.4 \pm 0.5$ & $43.2 \pm 0.5$ \\
\hline \multicolumn{3}{|l|}{ Lifestyle variables } \\
\hline \multicolumn{3}{|l|}{ Sexual-partner status — no./total no. (\%) } \\
\hline Partner & $1410 / 1551(90.9)$ & $1449 / 1565(92.6)$ \\
\hline No partner & $141 / 1551(9.1)$ & $116 / 1565(7.4)$ \\
\hline \multicolumn{3}{|l|}{ Smoking status - no./total no. (\%) } \\
\hline Never smoked & $472 / 1575(30.0)$ & $461 / 1577(29.2)$ \\
\hline Former smoker & $752 / 1575(47.7)$ & $785 / 1577(49.8)$ \\
\hline Current smoker & $351 / 1575(22.3)$ & $331 / 1577(21.0)$ \\
\hline \multicolumn{3}{|l|}{ Alcohol intake - no./total no. (\%) } \\
\hline None & $247 / 1600(15.4)$ & $260 / 1598(16.3)$ \\
\hline 1-4 days/wk & $970 / 1600(60.6)$ & $982 / 1598(61.5)$ \\
\hline$\geq 5$ days $/ w k$ & $383 / 1600(23.9)$ & $356 / 1598(22.3)$ \\
\hline
\end{tabular}




\begin{tabular}{|c|c|c|}
\hline Variable & $\begin{array}{c}\text { Training Set } \\
(\mathrm{N}=1610)\end{array}$ & $\begin{array}{l}\text { Validation Set } \\
\quad(N=1609)\end{array}$ \\
\hline \multicolumn{3}{|c|}{ Self-reported general health — no./total no. (\%) } \\
\hline Excellent or very good & $450 / 1578(28.5)$ & $443 / 1595(27.8)$ \\
\hline Good & $730 / 1578(46.3)$ & $733 / 1595(46.0)$ \\
\hline Fair or poor & $398 / 1578(25.2)$ & $419 / 1595(26.3)$ \\
\hline \multicolumn{3}{|l|}{ Body-mass index — no./total no. (\%) } \\
\hline$<25$ & $410 / 1576(26.0)$ & $419 / 1590(26.4)$ \\
\hline$\geq 25$ to $<30$ & $790 / 1576(50.1)$ & $774 / 1590(48.7)$ \\
\hline$\geq 30$ & $376 / 1576(23.9)$ & $397 / 1590(25.0)$ \\
\hline \multicolumn{3}{|l|}{ Coexisting illness - no./total no. (\%) $\mathbb{S}$} \\
\hline \multicolumn{3}{|l|}{ Number } \\
\hline 0 & $804 / 1588(50.6)$ & $780 / 1578(49.4)$ \\
\hline 1 & $414 / 1588(26.1)$ & $440 / 1578(27.9)$ \\
\hline$\geq 2$ & $370 / 1588(23.3)$ & $358 / 1578(22.7)$ \\
\hline \multicolumn{3}{|l|}{ Type } \\
\hline Heart disease & $272 / 1588(17.1)$ & $236 / 1578(15.0)$ \\
\hline Hypertension & $451 / 1588(28.4)$ & $444 / 1578(28.1)$ \\
\hline Chronic bronchitis or asthma & $120 / 1588(7.6)$ & $118 / 1578(7.5)$ \\
\hline Diabetes & $104 / 1588(6.5)$ & $132 / 1578(8.4)$ \\
\hline Prostate disease & $165 / 1588(10.4)$ & $156 / 1578(9.9)$ \\
\hline Cancer & $87 / 1609(5.4)$ & $83 / 1607$ (5.2) \\
\hline Stroke & $63 / 1609$ (3.9) & $48 / 1607(3.0)$ \\
\hline
\end{tabular}

* Plus-minus values are means \pm SE. The study population of 3219 men was subdivided into four 10 -year age groups (40-49 years, 50-59 years, 60-69 years, and 70-79 years) to preserve the age stratification of the study design. In each of the four decade samples $\left(\mathrm{S}_{i}\right)$, two data sets were randomly generated: a training set $\left(T_{i}\right)$ and a validation set $\left(V_{i}\right)$. To generate these data sets, each subject from $S_{i}$ was randomly assigned a unique value from a uniformly distributed random variable $U_{i}$ on the interval $[0$ to 1$]$. The samples were then sorted by $U_{i}$ into two halves; the first half was selected as the training random sample $\left(T_{i}\right)$, and the second half as the validation sample $\left(V_{i}\right)$. The four $T_{i}$ data sets were then combined to form a single training sample of 1610 subjects, and the four $V_{i}$ data sets were likewise combined to form a single validation sample of 1609 subjects. To convert the values for total testosterone to nanograms per milliliter, divide by 3.467. To convert the values for free testosterone to picograms per milliliter, divide by 3.467 . SF-36 denotes Medical Outcomes Study 36-Item Short-Form Health Survey.

$\uparrow$ The body-mass index is the weight in kilograms divided by the square of the height in meters.

$\checkmark$ In the entire analysis sample, the 2.5 th and 97.5 th percentiles were 7.3 and $30.6 \mathrm{nmol}$ per liter for total testosterone, 139.6 and $485.5 \mathrm{pmol}$ per liter for free testosterone, and 16.8 and $91.6 \mathrm{nmol}$ per liter for sex hormone-binding globulin.

$\int$ Coexisting illnesses included heart conditions, high blood pressure, stroke, cancer, bronchitis, asthma, peptic ulcer, epilepsy, diabetes, and diseases of the liver, kidney, and prostate.

Logistic regression with linear splines of testosterone as independent variables showed significantly increased odds ratios for sexual and physical symptoms below testosterone thresholds (Section 3 in the Supplementary Appendix). Thus, for the symptom of a decreased frequency in morning erection, a reduction of $1 \mathrm{nmol}$ per liter (0.29 ng per milliliter) in total testosterone below $11 \mathrm{nmol}$ per liter was associated with an odds ratio of 1.10 (95\% confidence interval [CI], 1.02 to 1.19 ), whereas no significant relationship with the symp- tom was observed at a total testosterone level above $11 \mathrm{nmol}$ per liter. This indicated a total testosterone threshold for poor morning erection at $11 \mathrm{nmol}$ per liter, with an apparent plateau effect above this threshold. Similarly, a total testosterone threshold was identified for a low frequency of sexual thoughts at $8.0 \mathrm{nmol}$ per liter and for erectile dysfunction at $8.5 \mathrm{nmol}$ per liter; a reduction of $1 \mathrm{nmol}$ per liter in total testosterone below $8 \mathrm{nmol}$ per liter was associated with an odds ratio of 1.48 (95\% CI, 1.20 to 1.83 ) for a low fre- 


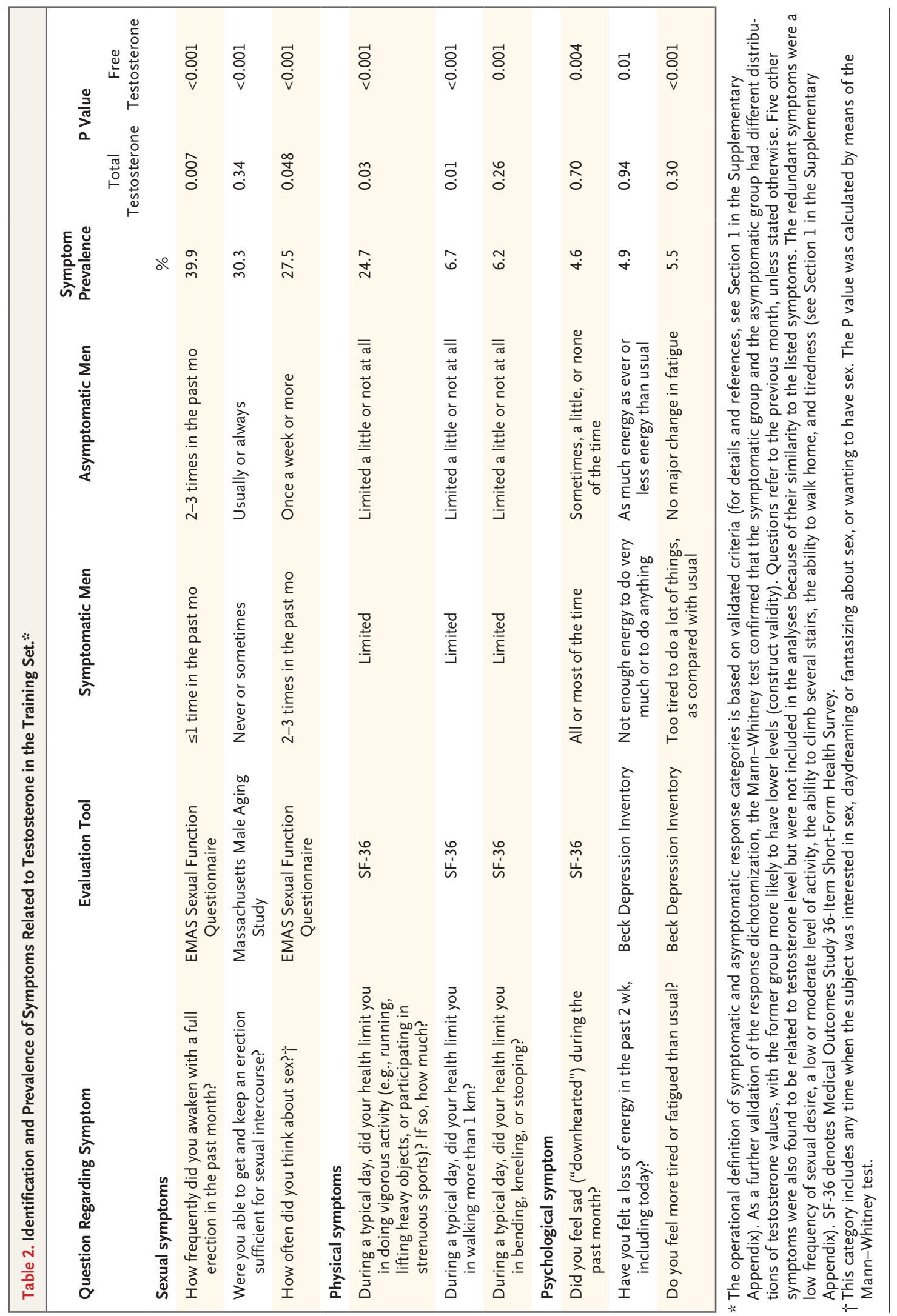




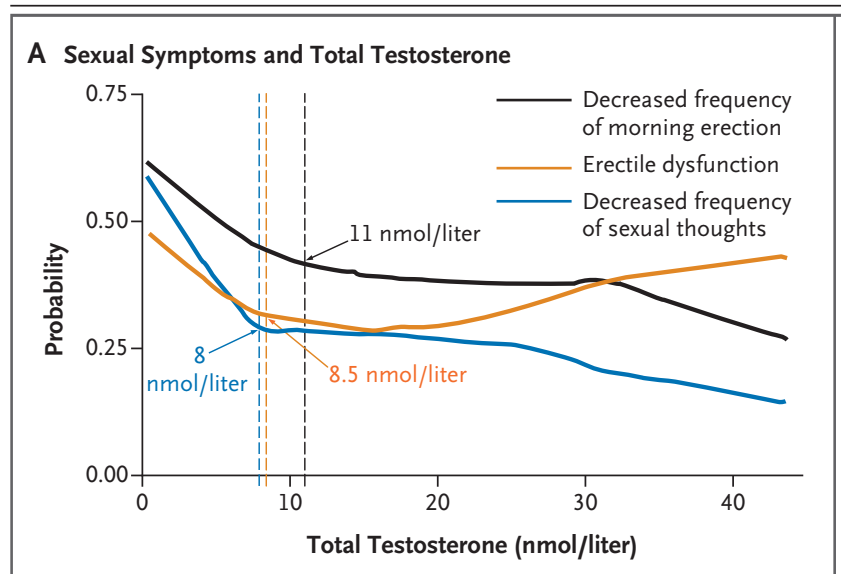

B Sexual Symptoms and Free Testosterone

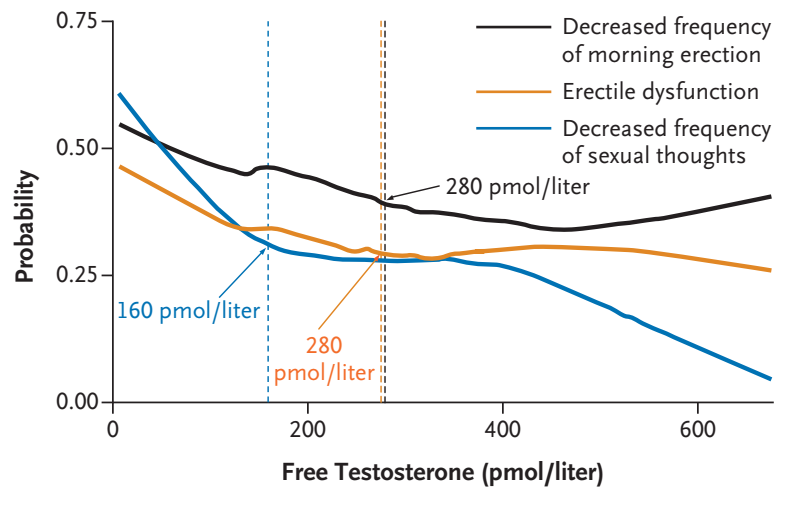

C Physical Symptoms and Total Testosterone

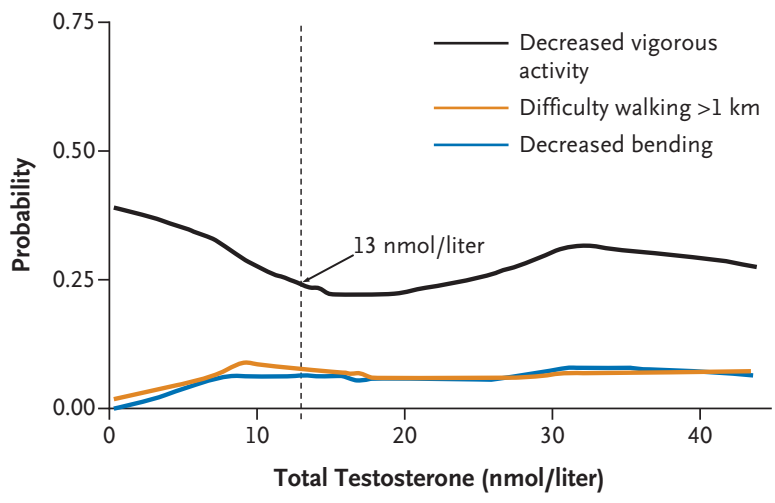

D Physical Symptoms and Free Testosterone

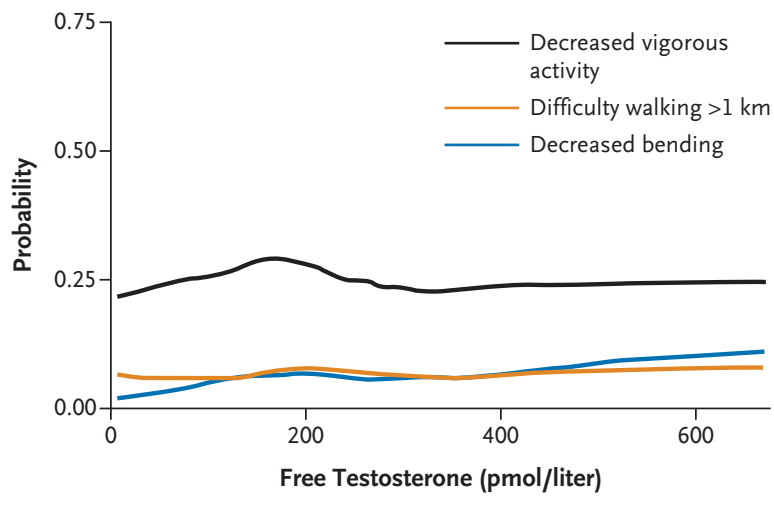

E Psychological Symptoms and Total Testosterone

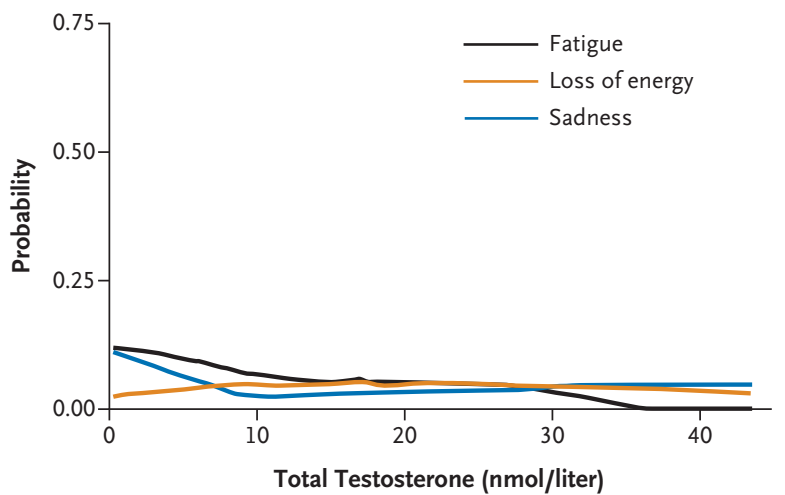

F Psychological Symptoms and Free Testosterone

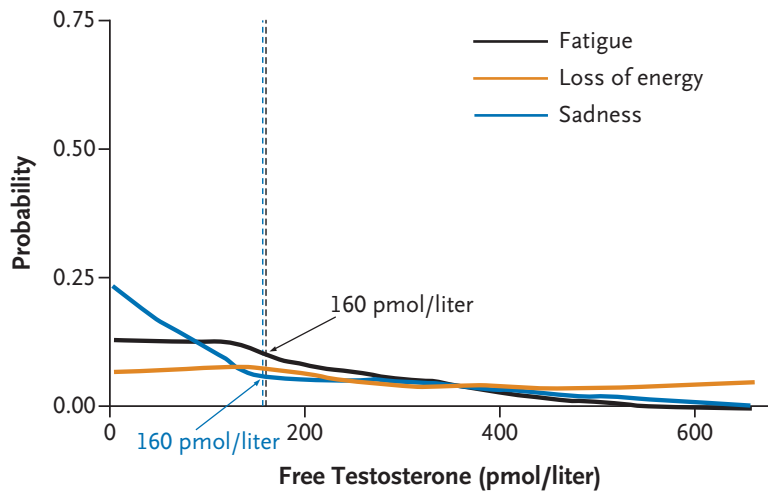

Figure 1. Probability of Symptoms on the Basis of Levels of Total Testosterone and Free Testosterone.

Shown is the age-adjusted probability of the presence of nine symptoms - including sexual symptoms (Panels A and B), physical symptoms (Panels C and D), and psychological symptoms (Panels E and F) - in men on the basis of individual levels of total testosterone and free testosterone. Threshold testosterone levels, indicated by vertical lines, are shown for symptoms for which the probability was significantly increased among men with a decreased testosterone level, as compared with those with an increased level of testosterone. No thresholds were identified for physical symptoms associated with free testosterone (Panel D) or for psychological symptoms associated with total testosterone (Panel E). All values were calculated with the use of locally weighted linear regression. To convert the values for total testosterone to nanograms per milliliter, divide by 3.467. To convert the values for free testosterone to picograms per milliliter, divide by 3.467 .

The New England Journal of Medicine 
quency of sexual thoughts, and such a reduction below $8.5 \mathrm{nmol}$ per liter was associated with an odds ratio of 1.23 (95\% CI, 1.06 to 1.42) for erectile dysfunction. The total testosterone threshold for a decreased level of vigorous activity was 13 nmol per liter; a reduction of $1 \mathrm{nmol}$ per liter in total testosterone below $13 \mathrm{nmol}$ per liter was associated with an odds ratio of 1.11 (95\% CI, 1.03 to 1.19). Free testosterone thresholds for a low frequency of sexual thoughts and for sadness were 160 pmol per liter, and the threshold for both erectile dysfunction and a decreased frequency of morning erection was 280 pmol per liter. The free testosterone threshold for an increased level of fatigue was $160 \mathrm{pmol}$ per liter (Section 3 in the Supplementary Appendix).

\section{CLUSTERING OF SYMPTOMS WITH TESTOSTERONE}

In the training set, multiple correspondence analyses delineated combinations of individual symptoms that were associated with one another and also with a low or normal testosterone level (Fig. 2A). Two distinct clusters could be identified. The absence of symptoms was clustered with a total testosterone level of at least $8.0 \mathrm{nmol}$ per liter, a total testosterone level of $11 \mathrm{nmol}$ per liter or more, and a free testosterone level of $220 \mathrm{pmol}$ per liter or more. The presence of the three sexual symptoms was clustered with a low total testosterone level ( $<8 \mathrm{nmol}$ per liter), a total testosterone level of less than $11 \mathrm{nmol}$ per liter, and a free testosterone level of less than 220 pmol per liter in syndromic associations. Symptoms that were not in close proximity were not considered part of the syndrome of symptomatic low testosterone. In the validation set, a very similar pattern was observed, confirming the association between low total and free testosterone levels and the three sexual symptoms (Fig. 2B).

\section{CRITERIA FOR HYPOGONADISM}

On the basis of criteria developed in the training set, stratification of the number of sexual symptoms according to various testosterone thresholds revealed a consistent inverse relationship, with an increased number of sexual symptoms associated with a higher odds ratio for a decreased threshold value for total testosterone $(8.0$ to $11.0 \mathrm{nmol}$ per liter). This association was further strengthened by the addition of free testosterone levels to the analysis, relationships that were confirmed in the validation set (Table 3). The boundaries within which symptoms were significantly associated
Figure 2 (facing page). Multiple Correspondence Analysis (MCA) Showing Associations between Symptoms and Levels of Total Testosterone and Free Testosterone in the Training and Validation Sets.

In this MCA plot, variables (including low or normal testosterone levels and the presence or absence of symptoms) are considered to be highly associated if they are at the same distance and in the same direction from the origin where the horizontal axis (axis 1) and the vertical axis (axis 2) cross in the training set (Panel A) and the validation set (Panel B). Thus, the clustering of the categories of the variables in close proximity to one another is indicative of a syndromic association, which is highlighted by red circles. The values along the axes are indexes of the strength of the association between variables. On axis 1 , the presence of symptoms has positive coordinates (to the right of the origin), as compared with the absence of symptoms, with negative coordinates (to the left of the origin). Axis 2 helps identify symptoms that are related to a low testosterone level. The red clusters indicate the presence of the three sexual symptoms, with coordinates similar to those of a low testosterone level. The blue clusters indicate the absence of symptoms, with coordinates similar to those of a normal testosterone level. In contrast, the three psychological symptoms and, to a lesser extent, the three physical symptoms are located far from coordinates for normal and low testosterone, indicating that these symptoms are unrelated or weakly related to the testosterone level. The cluster patterns of symptoms in relation to total or free testosterone levels in the training set are virtually identical to those in the validation set. To convert the values for total testosterone to nanograms per milliliter, divide by 3.467. To convert the values for free testosterone to picograms per milliliter, divide by 3.467 .

with a low testosterone level in the validation set were demarcated by the presence of three sexual symptoms and a total testosterone level of less than $11 \mathrm{nmol}$ per liter (odds ratio, 1.71; 95\% CI, 1.08 to 2.63). The addition of a threshold of less than 220 pmol per liter for free testosterone increased the odds ratio, as compared with the total testosterone level alone, especially for thresholds between 8.0 and $11 \mathrm{nmol}$ per liter. These limits can be regarded as evidence-based criteria that are necessary (but not sufficient without the ancillary general health information) for the diagnosis of late-onset hypogonadism. The association between the presence of three sexual symptoms and a low testosterone level was attenuated by adjustment for age, body-mass index, and the number of coexisting illnesses (Table 3).

\section{ESTIMATED PREVALENCE OF HYPOGONADISM}

In this analysis sample, $4.1 \%$ of subjects had a total testosterone level of less than $8.0 \mathrm{nmol}$ per 


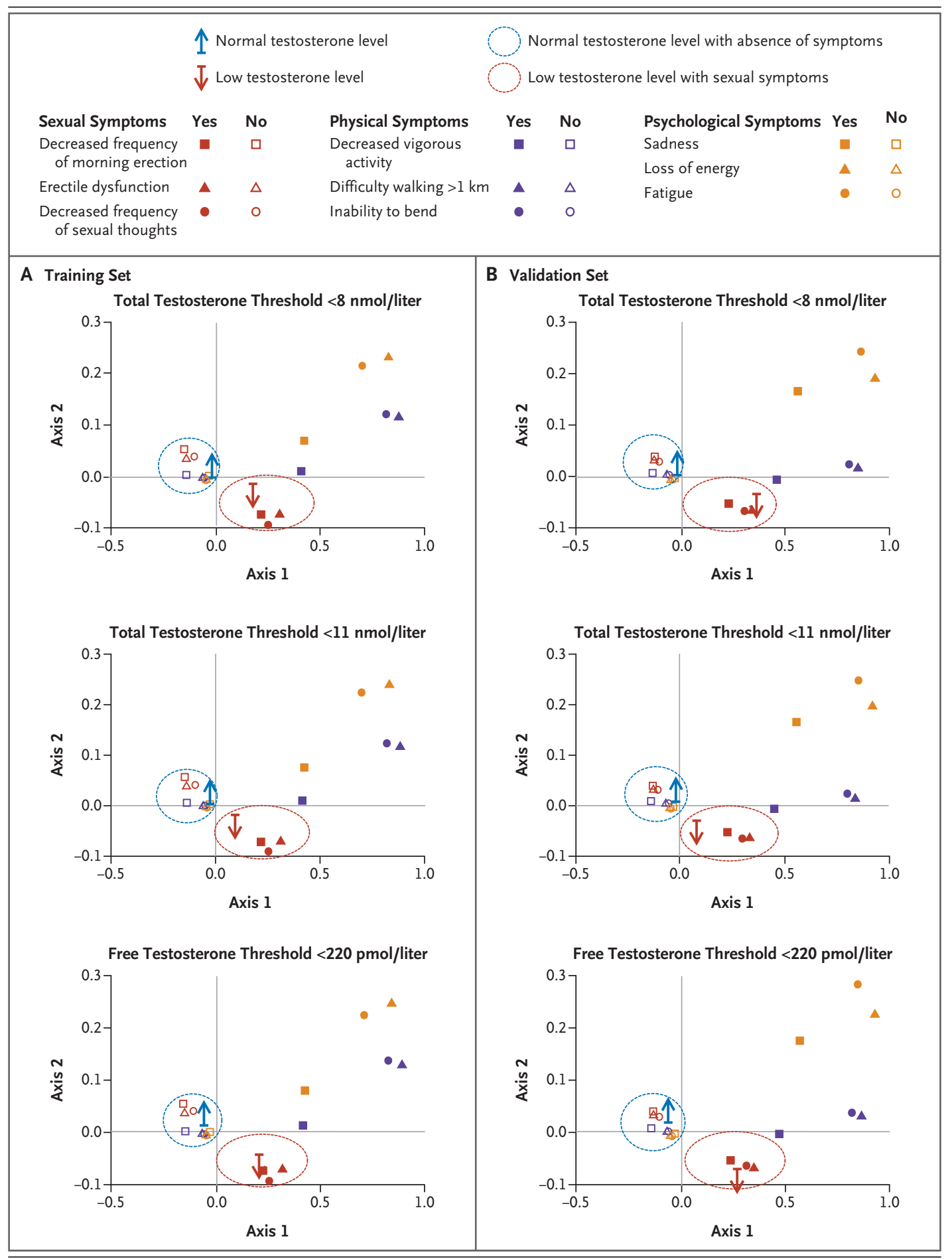

liter, and $17.0 \%$ had a total testosterone level of less than $11 \mathrm{nmol}$ per liter. If the syndrome of late-onset hypogonadism is considered to include at least three sexual symptoms associated with a total testosterone level of less than $11 \mathrm{nmol}$ per liter and a free testosterone level of less than 220

pmol per liter, among men for whom data were available for testosterone levels and questionnaire responses, the overall prevalence of late-onset hypogonadism in the EMAS study population would be $2.1 \%$ (63 of 2966 subjects) and would increase with age from $0.1 \%$ for men 40 to 49 years of age,

The New England Journal of Medicine 


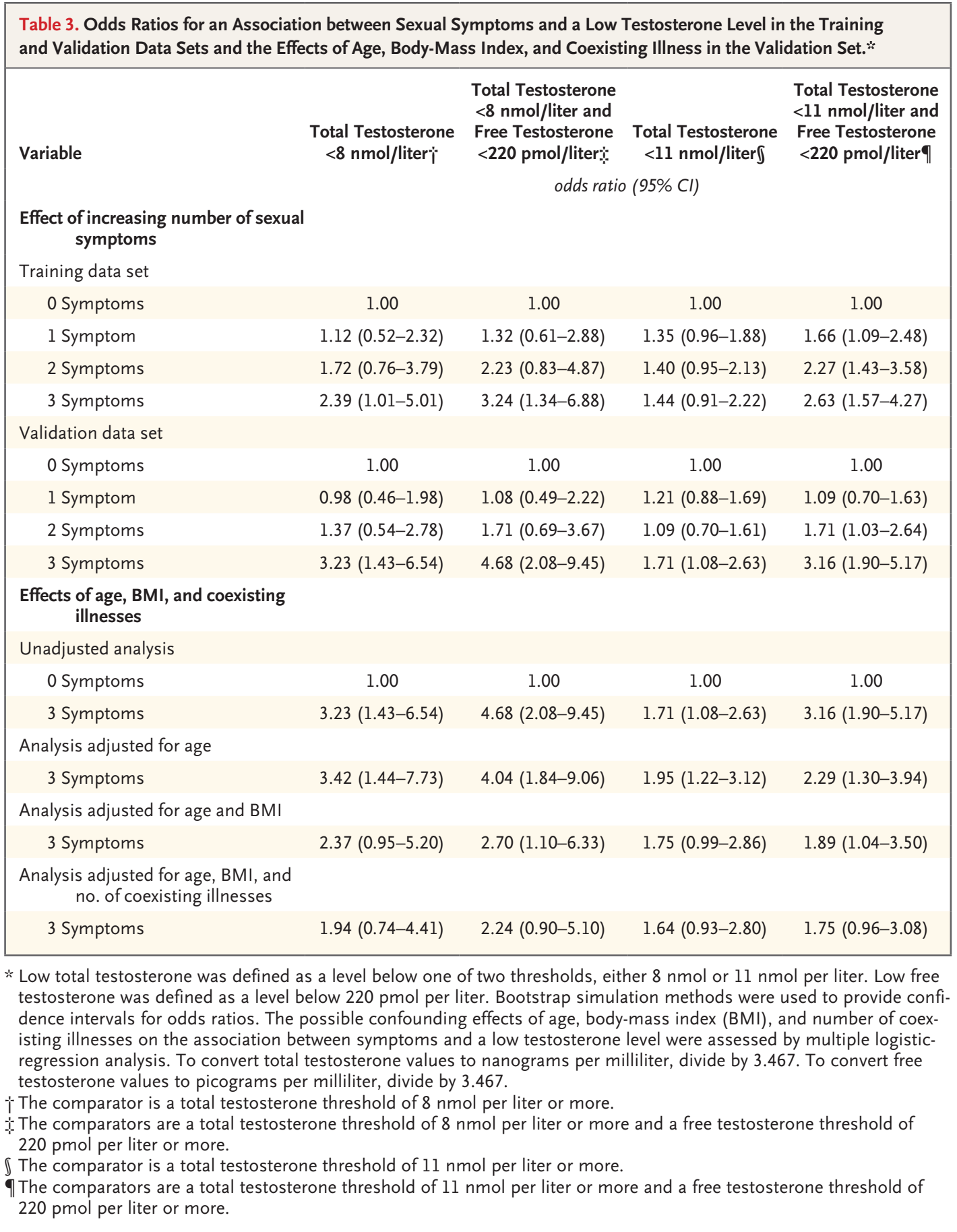

to $0.6 \%$ for those 50 to 59 years, to $3.2 \%$ for those 60 to 69 years, and to $5.1 \%$ for those 70 to 79 years (Fig. 2A in the Supplementary Appendix). The prevalence of late-onset hypogonadism would also rise with an increase in the body-mass index and an increasing number of coexisting illnesses (Fig. 2B and 2C in the Supplementary Appendix).

\section{DISCUSSION}

In this systematic investigation of a large, random sample of aging men from the general population, we used a reductive analytic approach to produce parsimonious clinical and biochemical criteria for diagnosing late-onset hypogonadism. 
We observed that many candidate symptoms of classic hypogonadism were not associated with decreased testosterone levels in older men. Even with the nine rigorously selected symptoms, differences in mean testosterone levels between symptomatic men and asymptomatic men were minimal, reflecting the weak overall association between symptoms and testosterone levels in this population (Fig. 1 in the Supplementary Appendix).

Further analyses revealed nonlinear threshold relationships between sexual symptoms and testosterone at levels around or below the lower limit of the eugonadal reference range for young men. Thus, sexual symptoms are of diagnostic importance in elderly men but should suggest androgen deficiency only when testosterone levels are clearly subnormal. ${ }^{26}$ Limited physical vigor was significantly associated with a low total testosterone level, with an apparent threshold at $13 \mathrm{nmol}$ per liter, an observation that is consistent with the finding in a previous study of elderly hypogonadal patients. ${ }^{22}$ Psychological symptoms had little or no association with the testosterone level, which supports the results of some studies 27,28 and contradicts the results of another. ${ }^{29}$ The presence of multiple symptom-specific testosterone thresholds supports the notion that different functional thresholds exist for the various androgen-dependent targets ${ }^{22,30,31}$ (Fig. 3A and 3B in the Supplementary Appendix).

A consistent syndromic clustering of three sexual symptoms with a low testosterone level can clearly be segregated from other, less germane symptoms and normal testosterone levels. These associations between multiple symptoms and testosterone thresholds were confirmed and augmented by the inverse dose-dependent relationship between an increasing number of sexual symptoms and decreasing threshold levels of testosterone. The boundaries for a significant association between symptoms and a low testosterone level identified the minimum criteria necessary to determine the syndrome of late-onset hypogonadism. Our suggested requirement of the presence of at least three sexual symptoms with a total testosterone level of less than $11 \mathrm{nmol}$ per liter and a free testosterone level of less than 220 pmol per liter supports recommendations in the latest practice guidelines. ${ }^{10,11}$ The influence of age, bodymass index, and number of coexisting illnesses on the association between symptoms and testosterone level and on the prevalence of late-onset hypogonadism is to be expected for a condition that develops with aging and is compatible with a multifactorial syndrome in which obesity and general health contribute to both a low testosterone level and symptoms. ${ }^{17,26}$

In our previously published work involving the EMAS cohort, in which we investigated the complex pathogenesis of the age-related decline in testosterone, different forms of putative biochemical hypogonadism (designated as primary, secondary, and compensated) could be distinguished in $23.3 \%$ of men with the use of only prespecified hormone criteria, without reference to clinical symptoms. ${ }^{32}$ This theoretical mechanistic information contrasts with the data provided by our current study, which is aimed at advancing practical clinical management. Thus, on the basis of the systematic determination of total and free testosterone thresholds below which symptoms became increasingly prevalent, the new data provide evidence-based criteria to define and facilitate correct diagnosis of the clinical syndrome of lateonset hypogonadism. Indeed, the prevalence of late-onset hypogonadism of $2.1 \%$, as determined in our current study, is much lower than that of biochemical hypogonadism. ${ }^{2,32}$ This finding underscores the paramount importance of using not only biochemical measures but also stringently defined, symptom-based criteria to prevent the overdiagnosis of late-onset hypogonadism.

The long list of nonspecific symptoms that have a potential association with testosterone deficiency makes it difficult to establish a diagnosis of lateonset hypogonadism. Thus, the prevalence of even the most specific sexual symptoms of androgen deficiency was relatively high among men with unequivocally normal testosterone levels, as reported in previous studies. ${ }^{33-35}$ This observation underscores the importance of specifying the presence of multiple (at least three) symptoms as a diagnostic criterion, preferably with the lower testosterone threshold ( $<8.0 \mathrm{nmol}$ per liter), to increase the probability of correctly diagnosing lateonset hypogonadism.

The addition of the measurement of free testosterone when the total testosterone level was under $8 \mathrm{nmol}$ per liter did not make a substantial difference with respect to the association between symptoms and testosterone level. This finding is similar to those in previous studies $22,33,36,37$ and supports the recommendation that total testosterone be used as the primary biochemical crite- 
rion for the diagnosis of late-onset hypogonadism. ${ }^{10,11,13}$ However, applying the threshold for free testosterone may be useful in patients with multiple symptoms and a borderline total testosterone level (8 to $11 \mathrm{nmol}$ per liter).

We used a current mass spectrometry-based method for measuring serum testosterone levels, as recommended by the Endocrine Society. ${ }^{38}$ Thus, the testosterone thresholds that we established should be applicable across laboratories with the use of a standard calibrator. However, in view of the known concern about the variability in and lack of accuracy of platform-based immunoassays, ${ }^{38}$ the proposed testosterone thresholds should be applied only with adjustments appropriate to the assay methods used in local laboratories.

Our study has certain limitations. The results are based on cross-sectional data from questionnaires and a single testosterone measurement in men from the general population. It is possible that the symptoms we selected had a higher specificity in symptomatic clinic patients. Our observations should be extrapolated to the diagnosis of testosterone deficiency in patients with the caveat that a low testosterone level requires confirmation with repeated measurement. Furthermore, the documentation of low testosterone levels in symptomatic elderly men does not invariably imply that a low testosterone level is the only or foremost cause of their symptoms. A comprehensive general assessment is required to seek potential alternative explanations. These data do not set the criteria for initiating testosterone replacement, for which clinical trials are required to determine the benefits and risks of treatment.

On the basis of data from the general population, our study indicated that the presence of three sexual symptoms combined with a total testosterone level of less than $11 \mathrm{nmol}$ per liter and a free testosterone level of less than 220 pmol per liter can be considered the minimum criteria for the diagnosis of late-onset hypogonadism in aging men. Our results also highlight the substantial overlap between late-onset hypogonadism and nonspecific symptoms of aging. The application of these new criteria can guard against the excessive diagnosis of hypogonadism and curb the injudicious use of testosterone therapy in older men.

Supported by a grant (QLK6-CT-2001-00258) from the Commission of the European Communities Fifth Framework Program Quality of Life and Management of Living Resources.

Presented in part at the 88th Annual Meeting of the Endocrine Society, Boston, June 24-27, 2006.

Disclosure forms provided by the authors are available with the full text of this article at NEJM.org.

We thank the men who participated in this study in eight countries; the research and nursing staff in the eight centers, C. Pott in Manchester, E. Wouters in Leuven, M. Nilsson in Malmö, M. del Mar Fernandez in Santiago de Compostela, M. Jedrzejowska in Lodz, H.-M. Tabo in Tartu, and A. Heredi in Szeged, for their meticulous data collection; C. Moseley in Manchester for data entry and project coordination; S. Goddard in Manchester for the preparation of the original figures; A. Araujo of the New England Research Institute for helpful discussions; the National Institute for Health Research Manchester Biomedical Research Centre; and Arthritis Research UK.

\section{APPENDIX}

The authors' affiliations are as follows: the Andrology Research Unit, Developmental and Regenerative Biomedicine Research Group (F.C.W.W., J.M.B., J.D.F.) and Arthritis Research UK Epidemiology Unit (A.T., S.R.P., T.W.O.), University of Manchester, Manchester Academic Health Science Centre, Manchester; Arthritis Research UK, Chesterfield (A.J.S.); the Department of Endocrinology, University College London, London (T.S.H.); the Department of Human Nutrition, University of Glasgow, Glasgow (M.E.J.L.); the School of Community Based Medicine, University of Manchester, Salford Royal NHS Trust, Salford (N.P.); and the Department of Surgery and Cancer, Imperial College London, Hammersmith Campus, London (I.T.H.) — all in the United Kingdom; the Department of Obstetrics, Gynaecology, and Andrology, Albert Szent-György Medical University, Szeged, Hungary (G.B.); the Department of Medicine, Santiago de Compostela University, Complejo Hospitalario Universitario de Santiago, Centro de Investigación Biomédica en Red de Fisiopatología Obesidad y Nutricion, Instituto Salud Carlos III, Santiago de Compostela, Spain (F.F.C.); the Endocrinology Unit, Department of Clinical Physiopathology, University of Florence, Florence, Italy (G.F.); the Reproductive Medicine Center, Skåne University Hospital, University of Lund, Malmö, Sweden (A.G.); the Department of Andrology and Reproductive Endocrinology, Medical University of Lodz, Lodz, Poland (K.K.); the Andrology Unit, United Laboratories of Tartu University Clinics, Tartu, Estonia (M.P.); the Departments of Geriatric Medicine (S.B.) and Andrology and Endocrinology (D.V.), Katholieke Universiteit Leuven, Leuven, Belgium; and the Laboratory of Molecular Endocrinology and Oncology, Laval University, Quebec, QC, Canada (F.L.).

The members of the EMAS study group are as follows: Florence, Italy: G. Forti, L. Petrone, G. Corona; Leuven, Belgium: D. Vanderschueren, S. Boonen, H. Borghs; Lodz, Poland: K. Kula, J. Slowikowska-Hilczer, R. Walczak-Jedrzejowska; London: I. Huhtaniemi; Malmö, Sweden: A. Giwercman; Manchester, United Kingdom: F. Wu, A. Silman, T. O’Neill, J. Finn, P. Steer, A. Tajar, D. Lee, S. Pye; Santiago de Compostela, Spain: F. Casanueva, M. Ocampo, M. Lage; Szeged, Hungary: G. Bartfai, I. Földesi, I. Fejes; Tartu, Estonia: M. Punab, P. Korrovitz; Turku, Finland: M. Jiang. 
1. Feldman HA, Longcope C, Derby CA, et al. Age trends in the level of serum testosterone and other hormones in middleaged men: longitudinal results from the Massachusetts Male Aging Study. J Clin Endocrinol Metab 2002;87:589-98.

2. Harman SM, Metter EJ, Tobin JD, Pearson J, Blackman MR. Longitudinal effects of aging on serum total and free testosterone levels in healthy men. J Clin Endocrinol Metab 2001;86:724-31.

3. Morley JE, Kaiser FE, Perry HM III, et al. Longitudinal changes in testosterone, luteinizing hormone, and follicle-stimulating hormone in healthy older men. Metabolism 1997;46:410-3.

4. Wu FC. Guideline for male testosterone therapy: a European perspective. J Clin Endocrinol Metab 2007;92:418-9.

5. Shames D, Gassman A, Handelsman $\mathrm{H}$. Guideline for male testosterone therapy: a regulatory perspective. J Clin Endocrinol Metab 2007;92:414-5.

6. Miner MM, Seftel AD. Testosterone and ageing: what have we learned since the Institute of Medicine report and what lies ahead? Int J Clin Pract 2007;61:622-32. 7. Bhasin S, Wu F. Making a diagnosis of androgen deficiency in adult men: what to do until all the facts are in? Nat Clin Pract Endocrinol Metab 2006;2:529.

8. McKinlay JB, Travison TG, Araujo AB, Kupelian V. Male menopause: time for a decent burial? Menopause 2007;14:973-5. 9. Morales A, Schulman CC, Tostain J, Wu FCW. Testosterone deficiency syndrome (TDS) needs to be named appropriately - the importance of accurate terminology. Eur Urol 2006;50:407-9.

10. Nieschlag E, Swerdloff R, Behre HM, et al. Investigation, treatment, and monitoring of late-onset hypogonadism in males: ISA, ISSAM, and EAU recommendations. J Androl 2006;27:135-7.

11. Bhasin S, Cunningham GR, Hayes FJ, et al. Testosterone therapy in adult men with androgen deficiency syndromes: an Endocrine Society clinical practice guideline. J Clin Endocrinol Metab 2006;91: 1995-2010.

12. Snyder PJ. Hypogonadism in elderly men - what to do until the evidence comes. N Engl J Med 2004;350:440-2.

13. Liverman CT, Blazer DG. Testosterone and aging: clinical research directions. Washington, DC: National Academies Press, 2004

14. Lee DM, O'Neill TW, Pye SR, et al. The European Male Ageing Study (EMAS): de- sign, methods and recruitment. Int J Androl 2009;32:11-24.

15. O'Connor DB, Corona G, Forti G, et al. Assessment of sexual health in aging men in Europe: development and validation of the European Male Ageing Study sexual function questionnaire. J Sex Med 2008;5: 1374-85.

16. Labrie F, Bélanger A, Bélanger $\mathrm{P}$, et al. Androgen glucuronides, instead of testosterone, as the new markers of androgenic activity in women. J Steroid Biochem Mol Biol 2006;99:182-8.

17. Wu FC, Tajar A, Pye SR, et al. Hypothalamic-pituitary-testicular axis disruptions in older men are differentially linked to age and modifiable risk factors: the European Male Aging Study. J Clin Endocrinol Metab 2008;93:2737-45.

18. Vermeulen A, Verdonck L, Kaufman JM. A critical evaluation of simple methods for the estimation of free testosterone in serum. J Clin Endocrinol Metab 1999; 84:3666-72

19. Heinemann LA, Saad F, Heinemann K, Thai DM. Can results of the Aging Males' Symptoms (AMS) scale predict those of screening scales for androgen deficiency? Aging Male 2004;7:211-8.

20. Araujo AB, O'Donnell AB, Brambilla $D J$, et al. Prevalence and incidence of androgen deficiency in middle-aged and older men: estimates from the Massachusetts Male Aging Study. J Clin Endocrinol Metab 2004;89:5920-6.

21. Morley JE, Perry HM III, Kevorkian RT, Patrick P. Comparison of screening questionnaires for the diagnosis of hypogonadism. Maturitas 2006;53:424-9.

22. Zitzmann M, Faber S, Nieschlag E. Association of specific symptoms and metabolic risks with serum testosterone in older men. J Clin Endocrinol Metab 2006;91:4335-43.

23. Cleveland WS. Robust locally weighted fitting and smoothing scatterplots. J Am Stat Assoc 1979;74:829-36.

24. Harrell FE Jr. Regression modeling strategies: with applications to linear models, logistic regression, and survival analysis. New York: Springer-Verlag, 2001.

25. Benzécri, J-P. Correspondence analysis handbook. New York: Marcel Dekker, 1992.

26. Travison TG, Morley JE, Araujo AB, O'Donnell AB, McKinlay JB. The relationship between libido and testosterone levels in aging men. J Clin Endocrinol Metab 2006;91:2509-13.

27. Delhez M, Hansenne M, Legros JJ.
Andropause and psychopathology: minor symptoms rather than pathological ones. Psychoneuroendocrinology 2003;28:863-74. 28. Tancredi A, Reginster JY, Schleich F, et al. Interest of the Androgen Deficiency in Aging Males (ADAM) questionnaire for the identification of hypogonadism in elderly community-dwelling male volunteers. Eur J Endocrinol 2004;151:355-60. 29. Barrett-Connor E, Von Mühlen DG, Kritz-Silverstein D. Bioavailable testosterone and depressed mood in older men: the Rancho Bernardo Study. J Clin Endocrinol Metab 1999;84:573-7.

30. Bhasin S, Woodhouse L, Casaburi R, et al. Older men are as responsive as young men to the anabolic effects of graded doses of testosterone on the skeletal muscle. J Clin Endocrinol Metab 2005;90:678-88.

31. Gray PB, Singh AB, Woodhouse LJ, et al. Dose-dependent effects of testosterone on sexual function, mood, and visuospatial cognition in older men. J Clin Endocrinol Metab 2005;90:3838-46.

32. Tajar A, Forti G, O'Neill TW, et al. Characteristics of secondary, primary, and compensated hypogonadism in aging men: evidence from the European Male Ageing Study. J Clin Endocrinol Metab 2010;95: 1810-8.

33. Araujo $A B$, Esche GR, Kupelian V, et al. Prevalence of symptomatic androgen deficiency in men. J Clin Endocrinol Metab 2007;92:4241-7.

34. Gladh YM, Rahgozar M, Hammar ML, Fredriksson MG, Spetz AC. Prevalence of symptoms possibly related to PADAM, in a Swedish population aged 55, 65 and 75 years. Maturitas 2005;50:161-6.

35. Travison TG, Araujo AB, Wruck LM, Kupelian V, O’Donnell AB, McKinlay JB. Assessment of screening evaluations is not straightforward. Maturitas 2006;54:305-8. 36. Kelleher S, Conway AJ, Handelsman DJ. Blood testosterone threshold for androgen deficiency symptoms. J Clin Endocrinol Metab 2004;89:3813-7.

37. Travison TG, Araujo AB, Kupelian V, O'Donnell AB, McKinlay JB. The relative contributions of aging, health, and lifestyle factors to serum testosterone decline in men. J Clin Endocrinol Metab 2007;92: 549-55.

38. Rosner W, Auchus RJ, Azziz R, Sluss PM, Raff H. Utility, limitations, and pitfalls in measuring testosterone: an Endocrine Society position statement. J Clin Endocrinol Metab 2007;92:405-13.

Copyright (c) 2010 Massachusetts Medical Society. 